\title{
Oublier nos archaïsmes pour penser autrement la régulation médicale demain
}

\author{
Forget Our Archaisms to Think otherwise the Medical Dispatching Tomorrow
}

\author{
F. Braun \\ (C) SFMU et Lavoisier SAS 2018
}

Tous les professionnels s'accordent à dire que notre système de santé est au bord du gouffre. Pour le président du Conseil national de l'ordre des médecins, "il faut agir maintenant » [1], pour le député Mesnier « le système de santé, inventé à partir de la fin des années cinquante, est à bout de souffle » [2], et Samu-Urgences de France proposait, il y a deux ans déjà, une nouvelle organisation des urgences [3].

Les symptômes de cette crise sans précédent s'étalent régulièrement dans la presse professionnelle mais maintenant aussi dans la presse « grand public », et le drame de la jeune Naomi [4] a braqué les projecteurs sur les urgences, partie immergée de l'iceberg des dysfonctionnements : crise dans les établissements hospitaliers pour personnes âgées dépendantes (EHPAD), mal-être des soignants, surcharge des services d'urgence, déserts médicaux, fermeture de services ou d'hôpitaux, manifestations et prises de position des élus... Au-delà de ces signes, apparaît aussi de plus en plus une crise de confiance de nos concitoyens envers le système de santé et plus récemment les urgences, crise habilement orchestrée par les uns et les autres.

L'étiologie retrouve des causes que nous partageons avec les autres pays industrialisés, d'autres qui nous sont propres.

- Le vieillissement de la population et son lien avec la dépendance et la précarité (20 années de vie gagnées en 70 ans) amènent ces «papy-ou plutôt mamy-boomers » à nécessiter plus de soins ;

- l'explosion des pathologies chroniques, conséquence directe des améliorations thérapeutiques (on sauve plus d'infarctus du myocarde, mais on crée plus d'insuffisance cardiaque), ne fait que commencer ;

- l'accessibilité aux informations médicales (qui n'est en rien la diffusion de la connaissance médicale) entraîne

\section{F. Braun $(\square)$}

Samu-Urgences de France,

103, boulevard Magenta,

F-75010 Paris, France

e-mail : francois.braun@sudf.fr une autonomie, certes relative, du patient qui se veut de plus en plus acteur de sa prise en charge. D'affligé (Moyen Âge) à malade (imaginaire ?), puis à patient (fin $\mathrm{du} \mathrm{XIX}^{\mathrm{e}}$ siècle), il est maintenant devenu usager de la santé : les mots ont leur importance!

- notre société, aussi, a son lot de responsabilités. L'immédiateté promue dans tout et partout s'est maintenant installée dans les soins : « on » veut un docteur, un scanner, une IRM... immédiatement, « on » y a droit sinon « on » porte plainte... Médecins, nous sommes aussi responsables de cette dérive en ne sachant pas résister aux demandes des patients.

Ces quatre points, il y en a certainement d'autres, sont responsables d'une augmentation des besoins. Encore faudrait-il qu'il y ait une augmentation de l'offre, or c'est précisément le contraire qui se produit.

- L'augmentation vertigineuse de nos capacités diagnostiques et thérapeutiques entraîne une concentration des plateaux techniques aboutissant à des hôpitaux de proximité avec peu de moyens et d'autres, dits de recours, totalement saturés. Les services d'urgence en sont le reflet quotidien avec leurs difficultés à hospitaliser un patient faute de lit d'aval, difficultés qui ne font que s'aggraver (No bed challenge [5]) face à des services hyperspécialisés ;

- la diminution du temps médical disponible est elle aussi flagrante : moins de médecins (même si le numerus clausus a augmenté, il faut attendre la fin de leur formation), des médecins submergés par des tâches administratives, et surtout des médecins qui exercent autrement, en ville comme à l'hôpital. Pour remplacer un médecin qui part à la retraite, il en faudrait aujourd'hui deux ou trois...

Nous aboutissons au constat, bien connu des urgentistes en médecine de catastrophe, d'une inadéquation entre les besoins et les moyens. Alors, comme en situation de crise, il convient de changer de paradigme, mais ici pour longtemps. S'il est évident qu'il n'est pas possible d'agir sur les besoins de santé, c'est donc bien sur les moyens qu'il faut 
faire porter tous nos efforts. Traiter les symptômes, le plus souvent isolément, retardera peut-être l'issue fatale, mais ne sauvera pas notre système de santé.

\section{Les urgences comme solution et non comme problème [6]}

Les services d'urgence, les Samu-Centre 15, sont à la croisée des chemins. Lieux de confrontation tectonique entre la plaque « ville » et la plaque « hôpital », nos services explosent sous des pressions souvent contraires. L'augmentation quasi exponentielle de leur activité en est l'expression la plus visible : 20 millions de passages aux urgences, 25 millions d'appels au Samu par an [7] !

La question du ou des numéro(s) d'urgence est à la mode. N'oublions jamais qu'aujourd'hui, si l'on s'intéresse aux appels dirigés vers le 15, le 18 et la 112, les Samu reçoivent directement les deux tiers de tous ces appels et $93 \%$ de la totalité concerne la santé. Le dogme archaïque du numéro unique a plus de 30 ans, une époque où les téléphones portables n'existaient pas...

Mais c'est bien connu, trop de gens ont recours aux urgences pour rien ! Cette fadaise, entendue depuis longtemps pour les services d'urgence, fait maintenant florès pour le Samu. C'est simple, il suffit d'interdire aux gens (les autres, jamais soi...) d'appeler le « 15 » ou d'aller aux urgences pour rien, ou encore de fermer des services d'urgence ! Pourquoi n'y a-t-on pas pensé avant ! Mais c'est étonnant, ceux qui prônent ces solutions sont les mêmes à défiler dans la rue lorsqu'un service d'urgence va être remplacé par des consultations non programmées... Cette sentence est fausse. Nos concitoyens n'ont pas recours aux urgences ou au Samu-Centre 15 pour rien, ils y ont recours, car ils ne trouvent pas d'autre solution pour répondre à leur problème de santé. Ils ont un besoin, mais il n'y a pas d'autre moyen en face. Bien entendu, beaucoup (trop) de patients se présentent aux urgences alors qu'ils pourraient être pris en charge par un médecin généraliste, et beaucoup trop de gens appellent le « 15 » alors qu'ils pourraient appeler leur médecin traitant. Mais ces derniers ne sont pas joignables, ou pas disponibles, car souvent débordés : c'est un défaut de moyens !

En médecine, la définition de l'urgence est bien connue : l'apparition brutale de signes, de symptômes qui font craindre à une personne ou à son entourage un problème de santé qui nécessite un avis médical urgent (ou devrait-on dire immédiat ?). Mais lorsque ces symptômes initialement non alarmants durent depuis des heures ou des jours et que vous n'arrivez pas à joindre un médecin, cela devient-il une urgence ? Nos concitoyens ont déjà répondu à cette question.

\section{Quand doit-on alors avoir recours aux urgences?}

En cas de douleur thoracique, chacun sait-il faire le tri entre une crise d'angoisse et un infarctus du myocarde? J'en ai fait l'expérience, en tant que patient, et même avec plus de 30 ans de métier j'ai eu un doute qui heureusement n'a duré que quelques dizaines de minutes avant qu'un confrère cardiologue me débouche l'artère coronaire responsable de cet infarctus. Doit-on imposer ce dilemme à nos concitoyens au risque de voir augmenter les décès par défaut de prise en charge à temps ? Qui va avoir le courage de leur imposer ce choix ? Penser que chaque patient peut toujours faire le bon choix est une imposture. Vouloir dissocier, en théorie, les urgences et les soins non programmés en est une autre.

Un médecin doit faire ce choix complexe à leur place, même s'il n'est pas non plus infaillible. Un médecin urgentiste, dont le métier consiste à qualifier la demande (identifier le réel besoin de soins), agir pour préserver la vie et la fonction et orienter le patient au bon endroit au bon moment, est certainement plus à même d'effectuer ce choix en urgence.

Initier et coordonner le parcours de soins en urgence doit redevenir notre métier tant les succès sur la prise en charge de patients victimes d'infarctus du myocarde, d'accident vasculaire cérébral, de traumatisme grave ne sont pas discutables et doivent être étendus à toutes les pathologies d'urgence. Ce que nous évaluons comme « non urgent» doit bénéficier d'un autre parcours de soins.

Quand initier ce parcours de soins en urgence est la vraie question qu'il faut se poser au-delà des débats stériles et dogmatiques sur les numéros d'appel.

- À l'entrée de l'hôpital, c'est-à-dire au service des urgences. C'est le choix fait par certains pays qui se sont dotés de plateformes d'appels d'urgence non médicalisées qui, pour tout appel relevant de la santé, envoient une ambulance qui réalise un transport vers l'hôpital le plus proche. C'est le principe du scoop and run ou pack and bag cher aux Anglo-Saxons et à leurs indéfectibles supporteurs. Les départs-réflexes des sapeurs-pompiers répondent à cette logique d'engager immédiatement, sans discussion, un véhicule de secours et d'assistance aux victimes (VSAV) lorsqu'un appel arrive au 18 pour une urgence « vitale ». Comment expliquer alors que six patients sur dix transportés par un VSAV ne bénéficient pas d'une hospitalisation après avoir été transportés aux urgences, mais retournent à domicile?

- Dès l'appel. C'est le choix fait par d'autres pays, dont la France, avec la mise en place de la régulation médicale des appels au sein des Samu-Centres 15. Le patient est alors, en fonction des moyens disponibles, orienté vers le professionnel le plus apte à le prendre en charge sans être systématiquement dirigé vers un service d'urgence. 
Un système est-il meilleur que l'autre ? Peut-être pas, et les publications scientifiques ne nous permettent pas de trancher, mais ne dissocions pas cette phase préhospitalière des suivantes. Toute modification de l'une a inexorablement des répercussions sur les autres. En effet, le système scoop and run s'appuie sur des services d'urgence trois fois plus gros et avec trois fois plus de personnels que nos services français, et les hôpitaux s'organisent autour des urgences et non des soins programmés réclamés par la sacro-sainte tarification à l'activité (T2A). Ce n'est qu'une simple constatation malheureusement ignorée des adeptes du tronçonnage du parcours de soins visant à ne s'occuper que de ce qui les intéresse en négligeant la prise en charge globale, élément essentiel de la guérison.

\section{Faut-il modifier notre système de soins ?}

Certainement, et les services médicaux d'urgence doivent accompagner cette évolution. Il faut arrêter de lancer comme une incantation que le patient doit être au centre du système de soins, il faut le faire. Pour les urgences et les soins non programmés, le choix qui s'offre à nos responsables politiques est simple :

- soit passer outre la régulation médicale préalable via une plateforme d'appels d'urgence " fourre-tout » et diriger tous les patients qui le demandent vers l'hôpital. Il convient alors d'augmenter considérablement la capacité d'accueil des services d'urgence et d'organiser tous les hôpitaux autour de leur service d'urgence ;

- soit moderniser la régulation médicale au sein d'une plateforme "santé " et renforcer la réponse de soins primaires, c'est-à-dire la capacité des médecins généralistes à prendre en charge ce qui ne relève pas de l'hôpital [2].

Samu-Urgences de France à fait son choix, non par corporatisme ou dogmatisme mais par pragmatisme [3]. Nous n'avons pas, aujourd'hui ni demain, la capacité ni les moyens de modifier « de la cave au grenier » notre organisation hospitalière d'autant que nous ne pouvons affirmer que ce serait plus efficace. Pourtant, il y a urgence ! Par contre, nous avons les moyens de moderniser notre organisation, de la rendre rapidement plus efficace, plus efficiente, plus opérationnelle pour répondre aux besoins de santé d'aujourd'hui et de demain. Ne ratons pas cette opportunité !

\section{Le médecin régulateur est le chef d'orchestre du parcours de soins en urgence}

Médecin impliqué quotidiennement dans la prise en charge des patients de sa spécialité, il est urgentiste ou généraliste le plus souvent, parfois pédiatre, toxicologue, psychiatre, en fonction des appels à prendre en charge. Des progrès sont indispensables, rapidement :

- toutes les composantes de la régulation médicale doivent être réunies sur la même plateforme hospitalière ;

- les assistants de régulation médicale (ARM) doivent bénéficier d'une formation adaptée ;

- le développement de nouveaux outils doit permettre d'améliorer la pertinence de la régulation médicale : visioconférence, récupération des données de santé, régulation médicale augmentée, géolocalisation des effecteurs, anticipation du parcours de soins, réseaux de télémédecine dans les EHPAD et dans les déserts médicaux ;

- la plateforme doit être certifiée (HAS) et répondre à des critères de qualité régulièrement évalués, et doit mettre en place une politique d'amélioration des pratiques ;

- les plateformes santé doivent être organisées en réseau et se suppléer l'une l'autre ;

- le système d'information national des Samu-Centre 15 (SISamu) doit être rapidement mis en œuvre [7] et permettre une interconnexion avec les plateformes de secours ;

Le chef d'orchestre n'est rien sans ses musiciens :

- les services d'urgence doivent être gradués et organisés en réseau ;

- il faut permettre aux médecins généralistes de réinvestir la continuité des soins afin d'apporter une réponse de premier recours après évaluation par la régulation médicale : maisons médicales, identification de plages de consultation libres ;

- les médecins spécialistes libéraux doivent participer à la continuité des soins (radiologues, cardiologues, biologistes) ;

- l'organisation hospitalière doit permettre l'admission directe dans le service adapté sans passer par les urgences comme c'est déjà le cas pour certaines filières (réanimation, cardiologie interventionnelle, urgences neurovasculaires);

- des services hospitaliers de médecine polyvalente doivent pouvoir répondre aux besoins de santé actuels et contribuer à fluidifier l'aval des services d'urgence ;

- une organisation nationale des transports sanitaires urgents doit remplacer le méli-mélo anarchique actuel.

Il reste bien sûr la nécessité de jouer ensemble, médecins de toutes les spécialités, organisations hospitalières, agences régionales de santé (ARS), unions régionales de professionnels de santé (URPS) et... patients.

Par cette organisation, résolument moderne et disposant de technologies de pointe, nous diminuerons immédiatement la pression sur les services d'urgence et replacerons bien le patient au centre du dispositif de soins urgents et non programmés. La crise est aujourd'hui quotidienne ! En situation sanitaire exceptionnelle, la place du Samu et de la régulation médicale a la même importance qu'au quotidien [8]. 


\section{Références}

1. Bouet P (2018) Santé : explosion programmée. Éditions de l'Observatoire, Paris

2. Mesnier T (2018) Assurer le premier accès aux soins. Organiser les soins non programmés dans les territoires. Rapport remis à madame la ministre des Solidarités et de la Santé. http://solidarites-sante. gouv.fr/IMG/pdf/rapport_snp_vf.pdf (Dernier accès le 28 mai 2018)

3. Samu-Urgences de France (2015) Livre blanc. Organiser la médecine d'urgence en France : un défi pour l'avenir. http://www.samuurgences-de-france.fr/medias/files/129/821/livre-blanc-sudf-151015. pdf (Dernier accès le 28 mai 2018)

4. Franceinfo (2018) Ce que l'on sait de l'histoire de Naomi Musenga morte après avoir tenté d'appeler à l'aide le Samu. https://www.francetvinfo.fr/sante/ce-que-l-on-sait-de-1-histoire-denaomi-musenga-morte-apres-avoir-tente-d-appeler-a-l-aide-le-samu_ 2743789.html (Dernier accès le 28 mai 2018)

5. Samu-Urgences de France (2018) No bed challenge. http://nbc. samu-urgences-de-france.fr/default/index/a-propos (Dernier accès le 27 mai 2018)

6. Riou B (2013) Les urgences : une solution et non un problème ! Ann Fr Med Urg 3:1-2

7. Agence française de la santé numérique (2016) Programme SI-Samu. http://esante.gouv.fr/services/programme-si-samu (Dernier accès le 27 mai 2018)

8. Nahon M, Poirot N, Marx JS, et al (2016) Retour d'expérience des attentats du 13 novembre 2015. La régulation médicale zonale. Ann Fr Med Urg 6:16-21 